\title{
THE USE OF EELS TO STUDY THE URBACH EDGE OF SILICA
}

\author{
S. C. Cheng, S. L. Schiefelbein, L. Moore, M. Pierson-Stull, S. Sen and C. Smith \\ Science and Technology, Corning Incorporated, Corning, NY 14831
}

Silica, with its large band gap $(\sim 8 \mathrm{eV})$ and high transmission in the ultraviolet, is the material of choice for optical components in photolithography systems employing $\mathrm{KrF}(248 \mathrm{~nm}, 5.0 \mathrm{eV})$ and $\operatorname{ArF}(193 \mathrm{~nm}, 6.4 \mathrm{eV})$ lasers. Improved transmission is desired to improve efficiency and extend the range of usable laser wavelengths (e.g. to $\mathrm{F}_{2}$ lasers which operate at $157 \mathrm{~nm}, 7.9 \mathrm{eV}$ ). It is well known that impurities influence transmission, and there is some evidence that transmission is influenced by structure [1], and that structure affects the Urbach edge [2]. In order to better understand the influence of structure, we undertook to study the relationships between the Urbach edge, transmission at $193 \mathrm{~nm}\left(\% \mathrm{~T}_{\mathrm{i}} @ 193 \mathrm{~nm}\right)$ and fictive temperature $\left(\mathrm{T}_{\mathrm{f}}\right)$, which is thought to be a measure of glass structure.

The Urbach edge is typically studied via measurements of the optical absorption coefficient $(\alpha)$. However, since absorption increases with energy, direct measurement of $\alpha$ above $\sim 8 \mathrm{eV}$ is very difficult, and a new method was needed to investigate the Urbach edge of silica. In this study, $\alpha$ in the energy range 0-40 eV was obtained from low loss EELS data by Kramers-Kronig analysis [3]. The EELS experiments were carried out using a $200 \mathrm{kV}$ cold field emission TEM (Hitachi HF-2000) equipped with a Gatan 666 parallel EELS spectrometer interfaced via the Gatan EL/P software to a PC. The microscope was set in image mode and no objective lens aperture was used (energy resolution $\sim 0.6 \mathrm{eV}$ ). Prior to the EELS experiment, specimens were examined on the JEM-2000FX microscope equipped with an Evex ultra-thin window X-ray detector.

Figure 1 is a raw EELS spectrum over the range $0-40 \mathrm{eV}$ taken from a silica sample. After removing the contributions of multiple scattering, a single scattering profile was obtained and is shown in Fig. 2. Next, the surface scattering contribution was removed and the Kramers-Kronig analysis was applied to yield $\alpha$ as a function of energy, as shown in Fig. 3. The band gap energy $\left(\mathrm{E}_{0}\right)$ can be estimated from the plot at the point, near $10 \mathrm{eV}$, where the slope changes abruptly. Because $\alpha$ of silica glasses can be expressed as an exponential function of energy E [4], the slope of the Urbach edge, $\mathrm{K}$, can be determined from the slope of the straight line in the graph of $\ln (\alpha) v s$. E, shown in Fig. 4. Applying this analysis to several groups of silica samples, it was found that $\mathrm{E}_{0}$ varied very little (less than 5\%), and no obvious relationship was observed between $\mathrm{E}_{0}$ and \% $\mathrm{T}$ @ $193 \mathrm{~nm}$. However, K appeared to be related to\% $\mathrm{T}_{\mathrm{i}} @ 193 \mathrm{~nm}$. As shown in Fig. 5, the samples with higher K have higher\% $\% \mathrm{~T}_{\mathrm{i}} @ 193 \mathrm{~nm}$. In order to investigate the relationship between $\mathrm{T}_{\mathrm{f}}$ and $\mathrm{K}$, four samples of F-doped silica with different $T_{f}$ were studied. The different $T_{f}$ were achieved by specific heat treatments and were measured by FT-IR transmission spectroscopy. The results suggest a relationship between $\mathrm{K}$ and $\mathrm{T}_{\mathrm{f}}$. As shown in Fig. 6, the samples with lower $\mathrm{T}_{\mathrm{f}}$ have higher $\mathrm{K}$.

In summary, EELS was successfully applied to the study of the Urbach edge of silica. The results support the idea that structure affects transmission, and suggest that transmission can be improved by achieving a more relaxed glass structure.

Acknowledgements

The authors gratefully acknowledge Drs. J.G. Zheng, S.Y. Li and V.P. Dravid for the use of their 
facilities at EPIC of Northwestern University.

References

1. H. Hosono, Y. Ikuta, T. Kinoshita, and M. Hirano, Phys. Rev. Lett. 87 (2001) 175501.

2. K. Saito and J. Ikushima, J. Appl. Phys. 91 (2002) 4886.

3. R. F. Egerton, Electron Energy-Loss Spectroscopy in the Electron Microscope, 2nd edition, Plenum Press, New York, (1996).

4. I. T. Godmanis, A. N. Trukhin, and K. Hubner, Phys. Stat. Sol. (b) 116 (1983) 279.
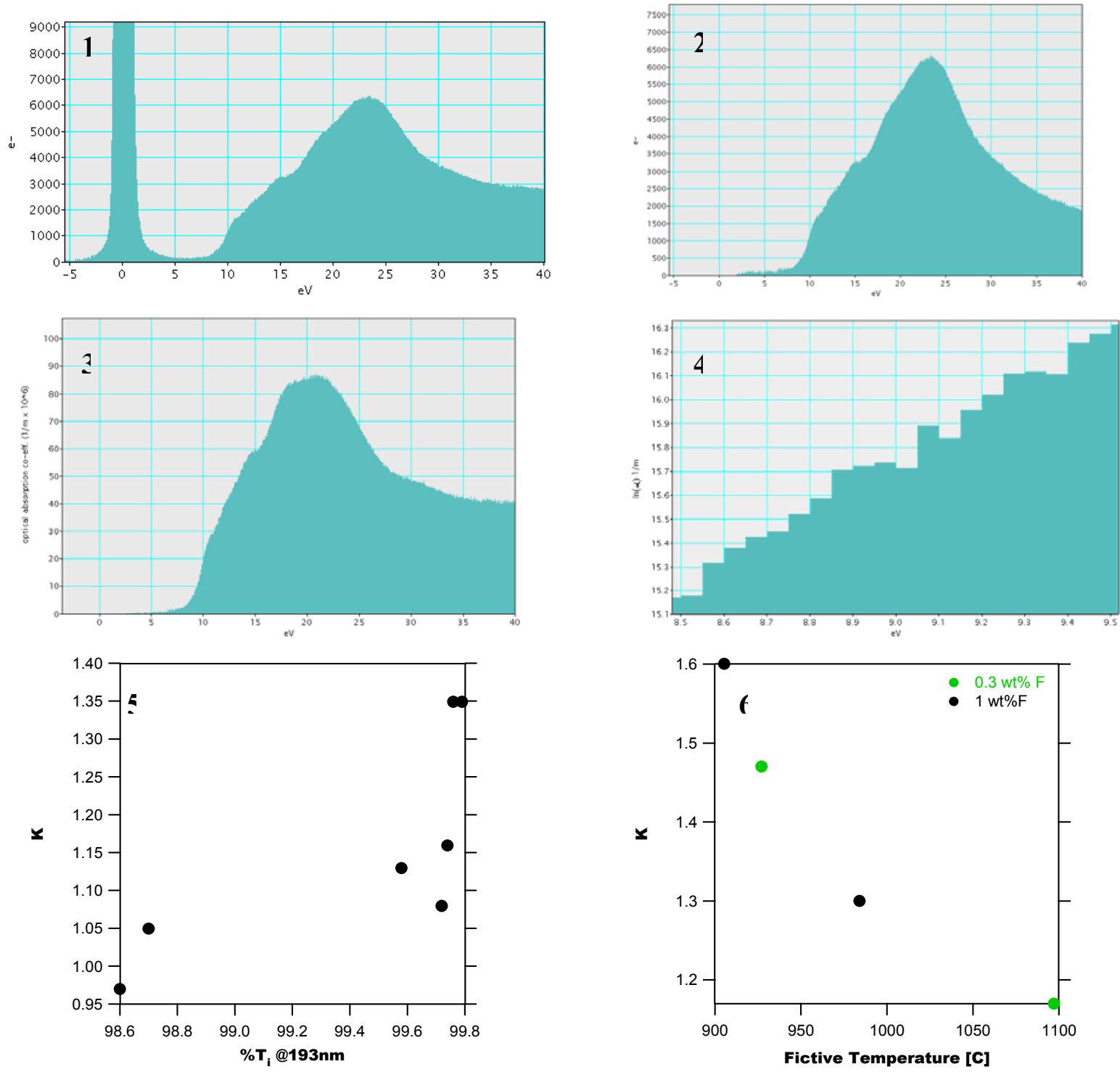

Fig. 1. A raw EELS spectrum taken from an example silica specimen.

Fig. 2. The single scattering profile obtained after Fourier-log deconvolution of the raw data.

Fig. 3. The optical absorption coefficient as a function of energy.

Fig. 4. The linear relationship between $\ln (\alpha)$ and $\mathrm{E}$ at energy range from $8.5 \mathrm{eV}$ to $9.5 \mathrm{eV}$.

Fig. 5. The correlation between the slope of the Urbach edge, K, and transmission @ $193 \mathrm{~nm}$.

Fig. 6 . The correlation between the slope of the Urbach edge, $\mathrm{K}$, and fictive temperature, $\mathrm{T}_{\mathrm{f}}$, for the four F-doped silica samples. 